\title{
Ajuste de modelos de platô de resposta via regressão isotônica
}

\author{
Response plateau models fitting via isotonic regression
}

\author{
Renata Pires Gonçalves ${ }^{I}$ Lucas Monteiro Chaves ${ }^{\text {II }}$ Taciana Villela SavianII \\ Fabyano Fonseca e Silva ${ }^{\text {IV }}$ Crysttian Arantes Paixãov
}

\section{RESUMO}

Dentro do contexto nutricional, a suplementação de microminerais em rações para aves frequentemente é feita em quantidades superiores às exigidas na tentativa de assegurar o bom desempenho dos animais. Os experimentos do tipo dose resposta são muito comuns na determinação de níveis ótimos dos nutrientes na ração e contemplam a utilização de modelos de regressão para atingir tal objetivo. Porém, na análise de regressão usual, geralmente, não se usa uma informação a priori sobre uma possível relação de ordem na variável resposta. A regressão isotônica é um método de estimação por mínimos quadrados que gera estimativas que satisfazem a mesma ordenação dos dados. Na teoria da regressão isotônica, essa informação é utilizada de forma essencial e espera-se que a eficiência do ajuste seja aumentada quando se faz uso dela. Diante do exposto, o presente trabalho tem como objetivo utilizar uma metodologia de regressão isotônica, como uma forma alternativa para analisar dados de deposição de zinco (Zn) na tíbia de aves machos da linhagem Hubbard. No estudo, foram considerados os modelos de platô de resposta polinomial quadrático e não linear exponencial. Além desses modelos, também foi proposto o ajuste de um modelo logarítmico para os dados e a eficiência da metodologia foi avaliada por meio de um estudo de simulação Monte Carlo, considerando diferentes cenários para os valores paramétricos. A isotonização dos dados propiciou uma melhora em todos os avaliadores de qualidade de ajuste considerados no trabalho. Dentre os modelos utilizados, o logarítmico apresentou estimativas dos parâmetros mais coerentes com os valores relatados na literatura, para os dados de deposição de Zn na tíbia de aves machos.
Palavras-chave: isotonização, modelos segmentados, modelos não lineares.

\section{ABSTRACT}

Within the nutritional context, the supplementation of microminerals in bird food is often made in quantities exceeding those required in the attempt to ensure the proper performance of the animals. The experiments of type dosage $x$ response are very common in the determination of levels of nutrients in optimal food balance and include the use of regression models to achieve this objective. Nevertheless, the regression analysis routine, generally, uses a priori information about a possible relationship between the response variable. The isotonic regression is a method of estimation by least squares that generates estimates which preserves data ordering. In the theory of isotonic regression this information is essential and it is expected to increase fitting efficiency. The objective of this work was to use an isotonic regression methodology, as an alternative way of analyzing data of $\mathrm{Zn}$ deposition in tibia of male birds of Hubbard lineage. We considered the models of plateau response of polynomial quadratic and linear exponential forms. In addition to these models, we also proposed the fitting of a logarithmic model to the data and the efficiency of the methodology was evaluated by Monte Carlo simulations, considering different scenarios for the parametric values. The isotonization of the data yielded an improvement in all the fitting quality parameters evaluated. Among the models used, the logarithmic presented estimates of the parameters more consistent with the values reported in literature.

Key words: isotonization, segmented models, nonlinear models.

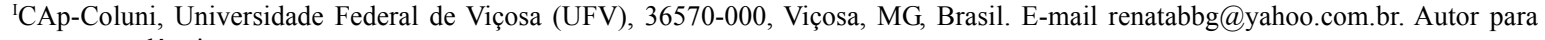
correspondência.

IIDepartamento de Ciências Exatas, Universidade Federal de Lavras (UFLA), Lavras, MG, Brasil.

IIIDepartamento de Ciências Exatas, Escola Superior de Agricultura Luiz de Queiroz (ESALQ), Universidade de São Paulo(USP), Piracicaba, SP, Brasil.

${ }^{\text {IV }}$ Departamento de Estatística, UFV, Viçosa, MG, Brasil.

vepartamento de Ciências Exatas, UFLA, Lavras, MG, Brasil.
} 


\section{INTRODUÇÃO}

A avicultura de corte é um dos segmentos da agropecuária que apresentou os maiores avanços tecnológicos. Esses avanços foram obtidos em diversas áreas, incluindo: a de melhoramento genético, manejo, sanidade e nutrição. Dentro do contexto nutricional, a suplementação de microminerais em rações para aves frequentemente é feita em quantidades superiores às exigidas na tentativa de assegurar o bom desempenho dos animais. Isso ocorre, geralmente, pelo desconhecimento do nutricionista quanto a real exigência das aves e esses excessos acarretam volumes excessivos de resíduos nas fezes, podendo ocasionar um desequilíbrio ambiental (GOMES et al., 2009).

O micromineral zinco $(\mathrm{Zn})$ está diretamente associado ao crescimento e ao desenvolvimento do tecido ósseo (UNDERWOOD \& SUTTLE, 1999), também atua como cofator ou componente de mais de 240 enzimas e sua deficiência pode acarretar problemas como anormalidades de pernas e dedos, além de reduzir o consumo de alimentos e, consequentemente, o desenvolvimento animal. Segundo o National Research Council - NRC (1994) e ROSTAGNO et al. (2005), as exigências de zinco para frangos de corte são de $40 \mathrm{e}$ $60 \mathrm{ppm}$, respectivamente, independentemente da idade e do sexo das aves.

Segundo COELHO et al. (1987), os experimentos delineados com a finalidade de avaliar exigências nutricionais de animais consideram, a priori, que a adição de um nutriente limitante a rações deficientes nesse nutriente e adequada nos demais resultará em crescimento linear do animal até que a exigência seja atendida, não ocorrendo respostas daí para frente. Os experimentos do tipo dose resposta são muito comuns na determinação de níveis ótimos dos nutrientes na ração e contemplam a utilização de modelos de regressão que podem ser lineares ou não em relação aos parâmetros.

O ajuste de modelos não-lineares, do ponto de vista metodológico, é bastante explorado por diversos autores nas mais diversas áreas, por meio das abordagens clássicas (SAVIAN et al., 2007a, 2007b; MENDES, et al., 2008; MAIA et al., 2009; ) e bayesiana (MUNIZ et al., 2007; MARTINS FILHO et al., 2008; SILVA et al., 2008; SAVIAN et al., 2009). Na análise de regressão usual, geralmente, não se usa uma informação a priori sobre uma possível relação de ordem na variável resposta. Na teoria da regressão isotônica, essa informação é utilizada de forma essencial e esperase que a eficiência do ajuste seja aumentada quando se faz uso dela.

O termo "isotônica" refere-se ao fato de a variável resposta aumentar com o aumento da variável independente. A técnica da regressão isotônica consiste em encontrar uma função não decrescente que minimize a soma de quadrados dos erros (BARLOW et al., 1972).

O presente trabalho tem como objetivo utilizar a metodologia de regressão isotônica, para analisar dados de deposição de zinco na tíbia de aves machos da linhagem Hubbard, ajustado aos modelos de platô de resposta polinomial quadrático e não linear exponencial. Também foi proposto o ajuste de um modelo de platô de resposta logarítmico.

A proposta de um modelo de platô de resposta logarítmico é bastante razoável, pois a função $\mathrm{y}=\ln (\mathrm{x})$ tem derivada $\mathrm{y}^{\prime}=1 / \mathrm{x}$ decrescente, isto é, quando $\mathrm{x}$ cresce a taxa de crescimento de y decresce. Dessa forma, no modelo de platô de resposta logarítmico, a passagem da fase crescente para a fase de resposta constante se dá de maneira mais suave, fato compatível com os fenômenos de saturação que ocorrem em fenômenos físicos.

\section{MATERIAL E MÉTODOS}

Os dados utilizados para o ajuste dos modelos, adaptados de REZENDE (2007), são provenientes de um experimento, conduzido nas instalações do Departamento de Zootecnia da UFRGS, instalado segundo um delineamento inteiramente casualizado com oito repetições. Os tratamentos estavam arranjados em uma estrutura fatorial $2 \times 2 \times 9$, sendo dois experimentos, dois sexos e nove doses de $Z n$ adicionadas na ração de aves da linhagem Hubbard. As doses de Zinco foram: 0, 15, 30, 45, 60, 75, 90, 105 e $120 \mathrm{ppm}$. As variáveis estudadas foram teor de $\mathrm{Zn}$ (em ppm) na tíbia e no dedo médio das aves. Para o presente trabalho, foram utilizados somente os dados referentes ao teor de $\mathrm{Zn}$ na tíbia de aves machos. Os dados de fêmeas não foram considerados devido ao fato de apresentarem um comportamento constante crescente que não justifica a utilização da regressão isotônica. A estimação dos parâmetros dos modelos não lineares: Modelo de platô de resposta polinomial quadrático (MPQ), Modelo de platô de resposta não linear exponencial (MNLE) e Modelo de platô de resposta logarítmico (MLOG), considerando os dados simulados e reais, foi feita pelo método de mínimos quadrados, sendo que a solução do sistema de equações normais não lineares foi obtida por meio do processo iterativo de Gauss-Newton (SOUZA, 1998).

Para ajustar os modelos de platô de resposta (MPQ, MNLE e MLOG), foi utilizada a função nls do pacote stats do software R (R Development Core Team, 
2010), apropriado para ajuste de funções não lineares. No processo de ajustamento, foram considerados os seguintes modelos:

Modelo de platô polinomial quadrático (MPQ):

$\mathrm{y}_{\mathrm{i}}=\mathrm{a}+\mathrm{bx}_{\mathrm{i}}+\mathrm{cx}^{2}{ }_{\mathrm{i}}+\varepsilon_{\mathrm{i}}$ se $\mathrm{x}_{\mathrm{i}}<\mathrm{x}_{0}$

$\mathrm{y}_{\mathrm{i}}=\mathrm{p}+\varepsilon_{\mathrm{i}}$ se $\mathrm{x}_{\mathrm{i}} \quad \mathrm{x}_{0}$

Modelo não-linear exponencial (MNLE):

$\mathrm{y}_{\mathrm{i}}=\mathrm{a} \exp \left(-\mathrm{c}\left(\mathrm{x}_{\mathrm{i}}-\mathrm{b}\right)^{2}\right)+\varepsilon_{\mathrm{i}}$ se $\mathrm{x}_{\mathrm{i}}<\mathrm{x}_{0}$

$\mathrm{y}_{\mathrm{i}}=\mathrm{p}+\varepsilon_{\mathrm{i}} \quad$ se $\mathrm{x}_{\mathrm{i}} \geq \mathrm{x}_{0}$

Modelo logarítmico (MLOG), proposto no presente trabalho:

$\mathrm{y}_{\mathrm{i}}=\mathrm{b} \log \left(\mathrm{x}_{\mathrm{i}}+\mathrm{a}\right)+\varepsilon_{\mathrm{i}}$ se $\mathrm{x}_{\mathrm{i}}<\mathrm{x}_{0}$

$\mathrm{y}_{\mathrm{i}}=\mathrm{p}+\varepsilon_{\mathrm{i}}$ se $\mathrm{x}_{\mathrm{i}} \geq \mathrm{x}_{0}$

em que: $\mathrm{y}_{\mathrm{i}}$ é o teor de zinco na tíbia do animal, em ppm;

$\mathrm{x}_{\mathrm{i}}$ é a i-ésima dose de zinco adicionada na ração, em ppm; a, b e c são parâmetros do modelo; $x_{0}$ é a dose de zinco na ração para a máxima deposição desse micromineral na tíbia dos animais; $p$ é o platô de resposta, ou seja, a máxima deposição de zinco na tíbia das aves e $\varepsilon_{\mathrm{i}}$ é o erro associado ao ajuste do modelo.

Para a regressão isotônica com pesos iguais, utilizou-se a função isoreg do pacote isotone, e, para a ponderada, ou seja, com pesos diferentes, utilizou-se a função cir.pava do pacote cir (R DEVELOPMENT CORE TEAM, 2010). Os pesos utilizados na ponderação, segundo a metodologia descrita em RODRIGUES et al. (2010), foram definidos como o inverso do módulo da diferença entre os dados originais e os dados obtidos pela regressão isotônica com pesos iguais.

A fim de avaliar a eficiência da regressão isotônica em estudos de exigência nutricional de aves, foi realizado um estudo de simulação de dados via técnica Monte Carlo. A simulação consistiu em gerar valores da variável resposta $\mathrm{y}_{\mathrm{i}}$ por meio da adoção de valores fixos para os parâmetros dos modelos, MPQ, MNLE e MLOG, considerando o erro aleatório proveniente de uma distribuição normal, com média zero e variância constante $\sigma^{2}$. O valor paramétrico para a variância foi definido em função do modelo segmentado utilizado para gerar os dados, ou seja, para o modelo MPQ, utilizou-se $\sigma^{2}=200 \mathrm{ppm}^{2}$ e $(\mathrm{a}=198$; $\mathrm{b}=1,5 ; \mathrm{c}=-0,01 ; \mathrm{x} 0=75 ; \mathrm{p}=254)$; para o modelo MNLE, $\mathrm{o}$ valor paramétrico foi $\sigma^{2}=130 \mathrm{ppm}^{2} \operatorname{com}(a=330 ; b=90$; $\mathrm{c}=0,00009 ; \mathrm{x} 0=90 ; \mathrm{p}=330)$ e; para o modelo MLOG, o valor utilizado foi $\sigma^{2}=60 \mathrm{ppm}^{2} \mathrm{e}(\mathrm{a}=30 ; \mathrm{b}=60 ; \mathrm{x} 0=65$; $\mathrm{p}=273$ ). Para cada modelo, foram geradas 1000 repetições de um conjunto com nove valores $y_{i}, i=1$, ..., 9, que representariam a deposição de zinco nos ossos, correspondentes a nove diferentes valores das doses de $\mathrm{Zn}\left(\mathrm{x}_{\mathrm{i}}\right)$ na ração, sendo $\mathrm{x}_{\mathrm{i}}=(0,15,30, \ldots, 120)$.

\section{RESULTADOS E DISCUSSÃO}

O procedimento de correção dos dados simulados via regressão isotônica, não ponderada $\mathrm{e}$ ponderada, mostrou-se eficiente em relação aos ajustes dos três modelos: MPQ, MNLE e MLOG. O procedimento reduziu o desvio padrão das estimativas para todos os parâmetros, implicando uma diminuição da amplitude dos intervalos de confiança. Verifica-se que a maior eficácia da isotonização foi constatada quando se utilizaram os modelos MNLE e MLOG. Tal eficiência foi evidente para a correção baseada na regressão isotônica não ponderada, em que as estimativas dos parâmetros $\left(\mathrm{x}_{0}, \mathrm{p}\right)$ foram: $(95,2 ; 330,8) \mathrm{e}$ $(66,94 ; 274,27)$, respectivamente, para os dois modelos (dados não apresentados nas tabelas).

Esse resultado está de acordo com RODRIGUES et al. (2010), que utilizaram uma transformação de dados via análise de regressão isotônica, para estudos de curvas de crescimento e concluíram que a metodologia possibilitou aumentar a porcentagem de convergência e menores erros quadráticos médios para os parâmetros dos modelos avaliados.

Do ponto de vista prático, verifica-se na figura 1(a) que os dados originais (não isotonizados) tendem a apresentar uma estabilização, ou seja, um platô (p) a partir da dose $60 \mathrm{ppm}$ de $\mathrm{Zn}$ na ração. Para os dados isotonizados ponderados, verifica-se que a estabilização ocorreu para dose $90 \mathrm{ppm}$, ou seja, a ponderação resultou em uma suavização demasiada da curva, o que acarretou em uma superestimação do parâmetro $x_{0}$. Para os dados isotonizados sem ponderação, a estabilização do platô ocorreu na dose 60ppm, coincidindo com o valor estimado para os dados originais. Por esse motivo, julga-se mais coerente considerar apenas a isotonização para o ajuste dos modelos (Figuras 1(a) e 1(b)). Esse comportamento pode ser visualizado numericamente por meio das estimativas do parâmetro $\mathrm{x}_{0}$ apresentadas nas tabelas $1,2 \mathrm{e} 3$.

Para o ajuste do modelo MPQ (Tabela 1), verifica-se que a isotonização não alterou de forma expressiva as estimativas dos parâmetros, exceto para o $\mathrm{x}_{0}$, em que se observa um aumento no valor desse parâmetro quando foi considerada a isotonização com ponderação. Verifica-se ainda nessa tabela, para todos os parâmetros do modelo, uma redução do desvio padrão das estimativas à medida que o procedimento de isotonização foi implementada, implicando uma diminuição da amplitude dos intervalos de confiança.

De forma semelhante ao ajuste do modelo MPQ, as estimativas dos parâmetros obtidas por meio 


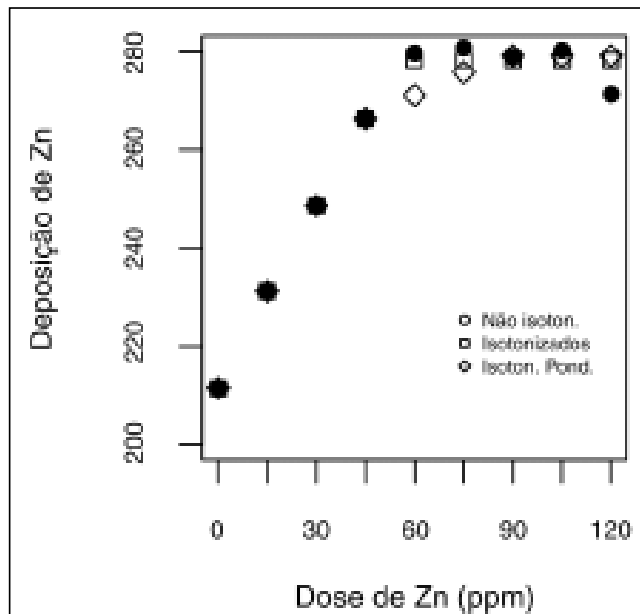

(a)

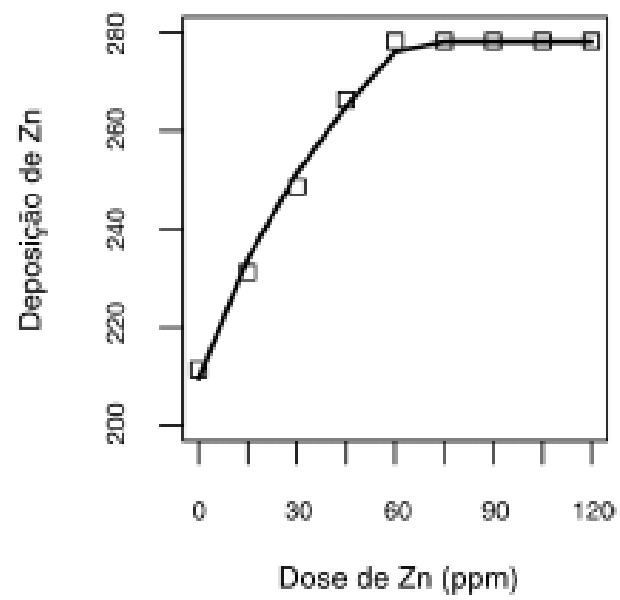

(b)

Figura 1 - Valores não isotonizados, isotonizados e isotonizados ponderados dos dados reais de deposição de zinco (Zn) na tíbia de aves (a) e ajuste do modelo logarítmico aos dados reais isotonizados (b).

do modelo MNLE (Tabela 2) também não sofreram alterações expressivas, exceto para o parâmetro $\mathrm{x}_{0}$ que foi superestimado à medida que o procedimento de isotonização foi implementado. Com relação à qualidade do ajuste desse modelo, nota-se uma redução expressiva no valor do desvio padrão residual quando foram consideradas as médias isotonizadas, sejam elas ponderadas ou não.

Tabela 1 - Estimativas dos parâmetros, com os respectivos desvios padrão (DP), limites (LI e LS) dos intervalos de $95 \%$ de confiança, coeficiente de determinação $\left(\mathrm{R}^{2}\right)$ e desvio padrão residual (DPR) para o ajuste do modelo polinomial quadrático (MPQ) às médias, não isotonizadas, isotonizadas e isotonizadas ponderadas, aplicadas aos dados reais de deposição de zinco (Zn) na tíbia de aves machos da linhagem Hubbard.

\begin{tabular}{|c|c|c|c|c|}
\hline Parâmetros & Estimativa & DP & LI & LS \\
\hline & \multicolumn{4}{|c|}{ 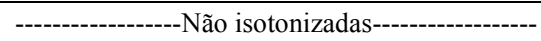 } \\
\hline $\mathrm{x}_{0}$ & 82,70 & 2,83 & 76,17 & 89,24 \\
\hline \multirow[t]{4}{*}{$\mathrm{P}$} & 280,86 & 0,91 & 278,77 & 282,95 \\
\hline & \multicolumn{4}{|c|}{$\mathrm{R}^{2}=0,992$} \\
\hline & \multicolumn{4}{|c|}{$\mathrm{DPR}=4,24$} \\
\hline & \multicolumn{4}{|c|}{---------------------Isotonizadas--------------------- } \\
\hline $\mathrm{x}_{0}$ & 80,91 & 2,54 & 75,04 & 86,77 \\
\hline \multirow[t]{4}{*}{$\mathrm{P}$} & 279,07 & 0,21 & 278,59 & 279,53 \\
\hline & \multicolumn{4}{|c|}{$\mathrm{R}^{2}=0,994$} \\
\hline & \multicolumn{4}{|c|}{$\mathrm{DPR}=2,34$} \\
\hline & \multicolumn{4}{|c|}{------------Isotonizadas ponderadas----------. } \\
\hline $\mathrm{x}_{0}$ & 86,74 & 2,19 & 81,69 & 91,78 \\
\hline \multirow[t]{3}{*}{$\mathrm{P}$} & 278,73 & 0,19 & 278,30 & 279,16 \\
\hline & \multicolumn{4}{|c|}{$\mathrm{R}^{2}=0,996$} \\
\hline & \multicolumn{4}{|c|}{$\mathrm{DPR}=1,76$} \\
\hline
\end{tabular}

Em relação ao modelo MLOG (Tabela 3), proposto neste trabalho, verifica-se que o processo de correção dos dados originais via regressão isotônica não alterou de maneira significativa as estimativas dos parâmetros, acarretando uma redução do desvio padrão das estimativas e implicando intervalos de confiança de menor amplitude, relativos a todos os parâmetros. Os índices de qualidade de ajuste, coeficiente de

Tabela 2 - Estimativas dos parâmetros, com os respectivos desvios padrão (DP), limites (LI e LS) dos intervalos de $95 \%$ de confiança, coeficiente de determinação $\left(\mathrm{R}^{2}\right)$ e desvio padrão residual (DPR) para o ajuste do modelo não linear exponencial (MNLE) às médias, não isotonizadas, isotonizadas e isotonizadas ponderadas, aplicadas aos dados reais de deposição de zinco $(\mathrm{Zn})$ na tíbia de aves machos da linhagem Hubbard.

\begin{tabular}{|c|c|c|c|c|}
\hline Parâmetros & Estimativa & DP & LI & LS \\
\hline & \multicolumn{4}{|c|}{ 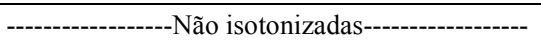 } \\
\hline $\mathrm{x}_{0}$ & 75,03 & 6,58 & 61,61 & 90,91 \\
\hline \multirow[t]{4}{*}{$\mathrm{P}$} & 278,50 & 1,91 & 273,99 & 283,18 \\
\hline & \multicolumn{4}{|c|}{$\mathrm{R}^{2}=0,982$} \\
\hline & \multicolumn{4}{|c|}{$\mathrm{DPR}=3,91$} \\
\hline & \multicolumn{4}{|c|}{ 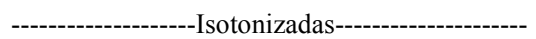 } \\
\hline $\mathrm{x}_{0}$ & 76,34 & 3,18 & 69,40 & 84,27 \\
\hline \multirow[t]{4}{*}{$\mathrm{P}$} & 278,72 & 0,92 & 276,55 & 280,98 \\
\hline & \multicolumn{4}{|c|}{$\mathrm{R}^{2}=0,996$} \\
\hline & \multicolumn{4}{|c|}{$\mathrm{DPR}=1,84$} \\
\hline & \multicolumn{4}{|c|}{------------Isotonizadas Ponderadas-----------' } \\
\hline $\mathrm{x}_{0}$ & 82,20 & 3,46 & 73,38 & 91,85 \\
\hline \multirow[t]{3}{*}{$\mathrm{P}$} & 278,58 & 0,95 & 276,23 & 281,04 \\
\hline & \multicolumn{4}{|c|}{$\mathrm{R}^{2}=0,996$} \\
\hline & \multicolumn{4}{|c|}{$\mathrm{DPR}=1,81$} \\
\hline
\end{tabular}

Ciência Rural, v.42, n.2, fev, 2012. 
Tabela 3 - Estimativas dos parâmetros, com os respectivos desvios padrão (DP), limites (LI e LS) dos intervalos de $95 \%$ de confiança, coeficiente de determinação $\left(\mathrm{R}^{2}\right)$ e desvio padrão residual (DPR) para o ajuste do modelo logarítmico (MLOG) às médias, não isotonizadas, isotonizadas e isotonizadas ponderadas, aplicadas aos dados reais de deposição de zinco $(\mathrm{Zn})$ na tíbia de aves machos da linhagem Hubbard.

\begin{tabular}{|c|c|c|c|c|}
\hline Parâmetros & Estimativa & $\mathrm{DP}$ & LI & LS \\
\hline & \multicolumn{4}{|c|}{------------------Não isotonizadas------------------ } \\
\hline $\mathrm{x}_{0}$ & 61,59 & 4,87 & 51,85 & 75,31 \\
\hline \multirow[t]{4}{*}{$\mathrm{P}$} & 280,05 & 0,91 & 277,95 & 282,15 \\
\hline & \multicolumn{4}{|c|}{$\mathrm{R}^{2}=0,993$} \\
\hline & \multicolumn{4}{|c|}{$\mathrm{DPR}=3,92$} \\
\hline & \multicolumn{4}{|c|}{----------------------Isotonizadas--------------------- } \\
\hline $\mathrm{x}_{0}$ & 63,19 & 2,69 & 57,22 & 70,25 \\
\hline \multirow[t]{4}{*}{$\mathrm{P}$} & 278,27 & 0,03 & 278,20 & 278,34 \\
\hline & \multicolumn{4}{|c|}{$\mathrm{R}^{2}=0,995$} \\
\hline & \multicolumn{4}{|c|}{$\mathrm{DPR}=2,09$} \\
\hline & \multicolumn{4}{|c|}{-------------Isotonizadas ponderadas------------ } \\
\hline $\mathrm{x}_{0}$ & 68,66 & 3,48 & 60,85 & 80,80 \\
\hline \multirow[t]{3}{*}{$\mathrm{P}$} & 278,11 & 0,34 & 277,33 & 278,90 \\
\hline & \multicolumn{4}{|c|}{$\mathrm{R}^{2}=0,993$} \\
\hline & \multicolumn{4}{|c|}{$\mathrm{DPR}=2,35$} \\
\hline
\end{tabular}

determinação e desvio padrão residual melhoraram tanto no caso isotonizado como nos isotonizados ponderados.

Considerando que, em experimentos do tipo dose resposta, em que a exigência nutricional dos animais é o foco principal do estudo, os parâmetros: $p$, que representa a deposição máxima de $\mathrm{Zn}$, e o parâmetro $\mathrm{x}_{0}$, que representa a dose de $\mathrm{Zn}$ na ração em que esse platô foi atingido, o modelo que apresentou o melhor ajuste aos dados experimentais foi o MLOG. Verifica-se, para o ajuste desse modelo, que o parâmetro $\mathrm{p}$ foi estimado com maior precisão, ou seja, sua estimativa apresentou o menor desvio padrão das estimativas quando comparado aos demais ajustes (Tabelas 1, 2 e 3), além da estimativa de $\mathrm{x}_{0}$ ter sido mais coerente com os dados experimentais (Figura 1(a)). Ressalta-se ainda que o modelo MLOG possui um parâmetro a menos quando comparado aos modelos MPQ e ao MNLE.

\section{CONCLUSÃO}

A metodologia de correção dos dados via análise de regressão isotônica, melhorou a qualidade dos ajustes dos modelos de platô de reposta, modelo polinomial quadrático e modelo não linear exponencial aos dados de deposição de zinco na tíbia de aves machos da linhagem Hubbard.
O modelo de platô de resposta logarítmico, ajustado aos dados reais de deposição de $\mathrm{Zn}$ na tíbia de machos da linhagem Hubbard, revelou-se eficiente na obtenção das estimativas do platô de resposta e sua abscissa.

\section{AGRADECIMENTO}

À Fundação de Amparo à Pesquisa do Estado de Minas Gerais (FAPEMIG), pela bolsa de estudo concedida, e aos revisores anônimos, pela valiosa contribuição.

\section{REFERÊNCIAS}

BARLOW, R.E. et al. Statistical inference under order restrictions: the theory and application of isotonic regression. New York: John Wiley \& Sons, 1972. 388p.

COELHO, L.S.S. et al. Modelos para estimar exigências nutricionais em suínos. Revista da Sociedade Brasileira de Zootecnia, v.16, n.1, p.102-110, 1987.

GOMES, P.C. et al. Níveis nutricionais de zinco para frangos de corte machos e fêmeas nas fases de crescimento e terminação. Revista Brasileira de Zootecnia, v.38, n.9, p.1719-1725, 2009. Disponível em $<$ http://www.scielo.br/scielo.php?script=sci_arttext\&pid=S151635982009000900011\&lng=en\&nrm=iso>. Acesso em: 01 fev. 2011. doi: 10.1590/S1516-35982009000900011.

MAIA, E. et al. Método de comparação de modelos de regressão não-lineares em bananeiras. Ciência Rural, v.39, n.5, p.13801386, 2009. Disponível em: <http://www.scielo.br/ scielo.php? script=s ci_arttext \& pid = S 0103 $84782009000500012 \& \operatorname{lng}=\mathrm{en} \& \mathrm{nrm}=\mathrm{iso}>$. Acesso em: 09 nov. 2010 .

MARTINS FILHO, S. et al. Abordagem Bayesiana das curvas de crescimento de duas cultivares de feijoeiro. Ciência Rural, v.38, n.6, p.1516-1521, 2008. Disponível em: $<\mathrm{http}$ / /www.scielo.br/scielo.php?script $=$ sci_arttext\&pid $=$ S0103$84782008000600004 \& \operatorname{lng}=$ en $\& n r m=i s o>$. Acesso em: 05 jun. 2010. doi: 10.1590/S0103-84782008000600004.

MENDES, P.N. et al. Modelo logístico difásico no estudo do crescimento de fêmeas da raça Hereford. Ciência Rural, v.38, n.7, p.1984-1990, 2008. Disponível em: <http:// www.scielo.br/scielo.php?script $=$ sci_arttext\&pid $=$ S010384782008000700029\&lng $=$ en $\& n r m=i s o>$. Acesso em: 05 jan. 2011. doi: 10.1590/S0103-84782008000700029.

MUNIZ, J.A. et al. Evaluation of lactation curve of lowyelding gir cows: a Bayesian approach. Acta Scientiarum. Animal Sciences, v.29, p.79-83, 2007.

NATIONAL RESEARCH COUNCIL - NRC. Nutrient requirements of poultry. 9.ed. Washington, D.C.: National Academy of Sciences, 1994. 155p.

R DEVELOPMENT CORE TEAM. R: a language and environment for statistical computing. Vienna, Austria: $R$ Foundation for Statistical Computing, 2010. ISBN 3-90005107-0. Disponível em: <http://www.R-project.org/>. Acesso em: 13 mar. 2010. 
REZENDE, D.M.L.C. et al. Ajuste de modelos de platô de resposta para a exigência de zinco em frangos de corte. Ciência e Agrotecnologia, v.31, n.2, p.468-478, 2007.

RODRIGUES, A. et al. Utilização da regressão isotônica em estudos de curvas de crescimento. Revista Brasileira de Biometria, v.28, n.4, p.85-101, 2010.

ROSTAGNO, H.S. et al. Tabelas brasileiras para aves e suínos: composição de alimentos e exigências nutricionais. Viçosa: UFV, 2005. 186p.

SAVIAN, T.V. et al. A study of in situ degradability: heterogeneity of variances and correlated errors. Scientia Agricola, v.64, p.548-554, 2007a.

SAVIAN, T.V. et al. Comparison of two mathematical models in the description of in situ degradability. Revista Brasileira de Ciência Veterinária, v.14, n.2, p.72-78, 2007b.
SAVIAN, T.V. et al. Análise bayesiana para modelos de degradabilidade ruminal. Ciência Rural, v.39, n.7, p.21692177, 2009. Disponível em <http://www.scielo.br/ s c ie lo.php ? s cript $=$ sci_art text \& pid $=$ S 0103 $84782009000700033 \& \operatorname{lng}=\mathrm{en} \& \mathrm{nrm}=\mathrm{iso}>$. Acesso em: 07 out. 2010. doi: 10.1590/S0103-84782009000700033.

SILVA, N.A. et al. Aplicação do método Bayesiano na estimação de curvas de crescimento de animais da raça Nelore. Revista Ceres, v.54, p.192-199, 2008.

SOUZA, G. da S. Introdução aos modelos de regressão linear e não linear. Brasília: Embrapa-SPI/Embrapa-SEA, 1998. 489p.

UNDERWOOD, E.J.; SUTTLE, N.F. The mineral nutrition of livestock. 3.ed. New York: CABI Publishing, 1999. 614p. 